\title{
Course of Ovarian Hyperstimulation Syndrome in 19 Intact Twin Pregnancies After Assisted Reproduction Techniques, With a Case Report of Severe Thromboembolism
}

\author{
Susanne Cupisti, Jasmin Emran, Andreas Mueller, Ralf Dittrich, Matthias W. Beckmann, and Helge Binder \\ Department of Obstetrics and Gynecology, University Hospital, Erlangen, Germany
}

$\mathrm{O}$ varian hyperstimulation syndrome (OHSS) is a serious complication of assisted reproduction techniques using in vitro fertilization and intracytoplasmic sperm injection (IVF/ICSI). Its etiology has still not yet been fully resolved. Human chorionic gonadotrophin, administered exogenously as well as produced endogenously during pregnancy, is responsible for the onset of OHSS, and high levels of estradiol appear to worsen the condition. In this case series, the course of mild to severe OHSS was evaluated in 19 intact twin pregnancies after IVF/ICSI. Another serious complication associated with OHSS is thromboembolic events. In these cases, the pregnancy can be protected through anticoagulation treatment, but there may be exceptions to this. This series includes a case of bilateral thrombosis of the internal and external jugular veins in the 7th gestational week in a twin pregnancy after OHSS and ICSI, with termination of the pregnancy in the 9th gestational week due to progressive thrombosis during anticoagulation therapy.

Forty per cent of children born after in vitro fertilization (IVF) or intracytoplasmic sperm injection (ICSI) are twins (Pinborg, 2005; Pinborg et al., 2004). The general consensus, with few exceptions, is that IVF/ICSI twins have neonatal outcomes (in terms of perinatal mortality, birthweight, gestational age, and small-for-gestational-age status) similar to those in twins conceived spontaneously (Pinborg, 2005; Pinborg et al., 2004). IVF/ICSI twins also have longterm outcomes similar to those in spontaneously conceived twins, with similar rates of neurological sequelae. However, twins conceived through IVF/ICSI techniques have considerably higher risks than IVF/ICSI singletons with regard to other short-term and long-term outcome measures (Pinborg, 2005; Pinborg et al., 2004).

Selective termination of pregnancy in IVF/ICSI twin pregnancies is usually performed for fetal abnormalities. In many countries, selective feticide in higher order multiple pregnancies is used to reduce the number of fetuses in order to maximize the pregnancy outcome of at least one healthy child (Pinborg, 2005; Pinborg et al., 2004). Induced abortion of a twin pregnancy because of maternal complications is fortunately rare.

There are no studies concerning only twin pregnancies conceived through IVF/ICSI with ovarian hyperstimulation syndrome (OHSS). Undoubtedly, the severity of OHSS increases in multiple pregnancies. Thromboembolic phenomena represent the most serious complications following assisted reproduction techniques. Deep vein thrombosis (DVT) in the upper extremities and jugular veins is a comparatively frequent complication (up to $10 \%$ ) after OHSS, but these thromboembolic events in OHSS - even during anticoagulation treatment - do not necessarily result in termination of the pregnancy.

This study presents data for 19 patients with viable twin pregnancies after IVF/ICSI who developed mild to severe OHSS. One of these was a pregnancy with late-onset OHSS after ICSI, complicated by DVT in the jugular vein in the 7 th gestational week. Progression of the thrombosis, despite high levels of anticoagulant drug treatment, led to termination of pregnancy in the 9 th gestational week.

\section{Materials and Methods}

A retrospective analysis was carried out with records of patients who presented in 2000 to 2005 with mild to severe ovarian hyperstimulation syndrome after receiving IVF/ICSI treatment. Data recorded included the patients' age, the duration of the hospital stay,

Received 13 June, 2006; accepted 14 July, 2006.

Address for correspondence: Dr Susanne Cupisti, Department of Obstetrics and Gynecology, Erlangen University Hospital, Universitätsstrasse 21-23, D-91054 Erlangen, Germany. E-mail: Susanne.Cupisti@gyn.imed.uni-erlangen.de 
type of OHSS (early, late; mild, moderate, severe), duration of stay in the intermediate care unit, complications (bleeding, thrombosis), family history of thromboembolism, symptoms of OHSS (abdominal pain, dyspnoea, oedema, ascites, nausea, elevated temperature), treatment strategies (dopamine, hydroxyethyl starch, mannitol, diuretics), and pregnancy outcome (mode of delivery, gestational week at delivery). Data for several serum parameters for OHSS on the day of admittance to the hospital were also compared with the data on the patient's day of discharge from hospital (blood group, hemoglobin, hematocrit, platelets, human chorionic gonadotrophin, estradiol, progesterone, sodium, potassium, total protein, albumin, chloride, glucose, creatinine, protein, albumin, C-reactive protein, partial thromboplastin time, aspartate aminotransferase, alanine aminotransferase, and gamma-glutamyl transferase). The data are presented as means plus or minus standard deviation; Student's $t$ test was used to assess the significance of all of the serological parameters studied.

\section{Results}

Of 174 patients with OHSS, 19 (10.9\%) had twin pregnancies. These patients were 29 to 37 years of age, and the duration of their hospital stays ranged from 4 to 33 days. Six patients $(31.6 \%)$ had earlyonset OHSS, whereas $13(68.4 \%)$ presented with late-onset OHSS. The time from embryo transfer to hospital admission ranged from 2 to 26 days. Thirteen patients were admitted to the intermediate care unit for up to 15 days (Table 1).

All patients, except one with mild OHSS, were treated with low-molecular-weight heparin (LMWH) and intravenous diuretics. Only one patient received mannitol. All but one of the patients had ascites; five $(26.3 \%)$ required drainage of the ascites using a pigtail catheter. Eleven patients $(57.9 \%)$ had pleural involvement - four $(21.0 \%)$ with unilateral pleural effusion and seven with bilateral pleural effusion $(36.8 \%)$. Ten patients $(52.6 \%)$ experienced dyspnoea,

\section{Table 1}

Comparison of Parameters in Relation to the Severity of Ovarian Hyperstimulation Syndrome in 19 Patients

\begin{tabular}{lccc}
\hline & I & II & III \\
\hline Patients & $1(5.3 \%)$ & $7(36.9 \%)$ & $11(57.9 \%)$ \\
Age (years) & 29 & $30-37$ & $27-41$ \\
Stay in hospital (days) & 4 & $6-12$ & $11-33$ \\
Early onset OHSS & 0 & $2(10.5 \%)$ & $4(21.0 \%)$ \\
Late onset OHSS & $1(5.2 \%)$ & $5(26.3 \%)$ & $7(36.8 \%)$ \\
Time from embryo transfer (days) & 26 & $9-14$ & $2-20$ \\
Intermediate care (patients) & 0 & $4(21.0 \%)$ & $9(47.3 \%)$ \\
Intermediate care (days) & 0 & 2.7 & 5.3 \\
\hline
\end{tabular}

Note: $\mathrm{OHSS}=$ ovarian hyperstimulation syndrome .

I = mild OHSS; II = moderate OHSS; III = severe OHSS.

\section{Table 2}

Diagnostic and Therapeutic Parameters in Relation to the Severity of Ovarian Hyperstimulation Syndrome in 19 Patients

\begin{tabular}{lllllll}
\hline & \multicolumn{1}{c}{ I } & \multicolumn{2}{c}{ II } & \multicolumn{3}{c}{ III } \\
\cline { 2 - 7 } & $N$ & $\%$ & $n$ & $\%$ & $n$ & $\%$ \\
\hline Dopamine & - & & 2 & 10.5 & 5 & 26.3 \\
Furosemide & - & & 2 & 10.5 & 9 & 47.3 \\
Ascites drainage & - & & 1 & 5.3 & 4 & 21.0 \\
Unilateral pleural effusion & - & & - & & 4 & 21.0 \\
Bilateral pleural effusion & - & & 3 & 15.8 & 4 & 21.0 \\
Abdominal pain & - & & 3 & 15.8 & 11 & 57.9 \\
Dyspnoea & - & 3 & 15.8 & 7 & 36.8 \\
Nausea & - & & 3 & 15.8 & 7 & 36.8 \\
Oedema & - & & - & & 3 & 15.8 \\
Temperature $>37^{\circ} \mathrm{C}$ & - & & 4 & 21.0 & 10 & 52.6 \\
$\quad$ (patients) & & & & & & \\
Temperature $>37^{\circ} \mathrm{C}$ & - & & & $1-5$ & & $2-14$ \\
$\quad$ (days) & & & & & & \\
\hline
\end{tabular}

Note: $\mathrm{OHSS}=$ ovarian hyperstimulation syndrome.

$\mathrm{I}$ = mild $\mathrm{OHSS}$; II = moderate $\mathrm{OHSS}$; III = severe OHSS

$14(73.6 \%)$ abdominal pain, $10(52.6 \%)$ nausea, and three patients $(15.8 \%)$ developed generalized oedema. Temperatures above $37{ }^{\circ} \mathrm{C}$ were recorded in 14 patients $(73.6 \%)$, and persisted for 1 to 14 days (Table 2).

The course of the values for the leukocyte count, hemoglobin, hematocrit, and creatinine showed significant differences between the day of admission and the day of discharge; however, no significant differences were observed between the admission and discharge values for platelets, human chorionic gonadotrophin (hCG), estradiol, progesterone, total protein, C-reactive protein (CRP), or liver enzymes (Table 3 ).

Seven patients $(36.8 \%)$ had preterm deliveries, six patients $(31.5 \%)$ had cesarean sections, and four patients $(21.0 \%)$ had preterm delivery by cesarean section (Table 4). Two patients, both with severe OHSS, developed complications due to bleeding. Two patients $(10.5 \%$; one with moderate OHSS and one with severe OHSS) reported a family history of thromboembolism. The patient with severe OHSS had a jugular vein thrombosis, and the pregnancy was terminated due to worsening problems (see the case report below).

\section{Case Report}

A 35-year-old patient presented with severe late-onset OHSS 12 days after oocyte retrieval and 10 days after the transfer of two embryos. She had a history of secondary infertility due to male subfertility and had undergone five ICSI treatments within 2 years, with one miscarriage and one living child born without OHSS developing.

The patient presented with abdominal pain, nausea, and mild shortness of breath. She had an uneventful medical history, but there was a family 
Table 3

Serological Parameters on the Day of Admittance (A) in Comparison with the Day of Discharge (D) in 19 Patients with Ovarian Hyperstimulation Syndrome

\begin{tabular}{|c|c|c|c|c|c|c|c|c|}
\hline & \multicolumn{2}{|c|}{ Leukocytes ( $\mu \mathrm{L})$} & \multicolumn{2}{|c|}{ Hemoglobin (dL) } & \multicolumn{2}{|c|}{ Hematocrit (\%) } & \multicolumn{2}{|c|}{ Creatinine (mg/dL) } \\
\hline & Admission & Discharge & Admission & Discharge & Admission & Discharge & Admission & Discharge \\
\hline OHSS I & 8.800 & 9.200 & 12.7 & 13.6 & 36 & 39 & 1.24 & 0.62 \\
\hline \multirow[t]{7}{*}{ OHSS II } & 17.900 & 17.200 & 14.6 & 12.6 & 42 & 37 & 0.62 & 0.54 \\
\hline & 14.200 & 11.500 & 15.4 & 13.2 & 44.2 & 37 & 1 & 0.7 \\
\hline & 13.700 & 6.300 & 14.8 & 11.8 & 42.3 & 33.8 & 1 & 0.78 \\
\hline & 15.400 & 8.210 & 17.4 & 11.4 & 51.2 & 33.7 & 0.74 & 0.69 \\
\hline & 13.100 & 9.970 & 14.5 & 12 & 44.1 & 34.8 & 0.83 & $\mathrm{x}$ \\
\hline & 23.400 & 11.000 & 17.2 & 12.3 & 47.8 & 35.9 & 0.94 & 0.67 \\
\hline & 16.200 & 10.400 & 16.1 & 12 & 47.3 & 35 & 0.78 & 0.62 \\
\hline \multirow[t]{9}{*}{ OHSS III } & 16.300 & 12.600 & 14.7 & 11.5 & 42 & 34 & 0.8 & 0.54 \\
\hline & 9.600 & 8.100 & 13.5 & 12.4 & 39.6 & 36 & 0.66 & 0.56 \\
\hline & 12.500 & 12.700 & 13.3 & 11.5 & 37.7 & 33 & 0.62 & 0.44 \\
\hline & 29.800 & 9.500 & 18.1 & 10.3 & 49.8 & 28.9 & 1.05 & 0.56 \\
\hline & 21.800 & 8.200 & 15.3 & 11.5 & 42.9 & 35 & $\mathrm{x}$ & $\mathrm{x}$ \\
\hline & 15.000 & 10.300 & 16.8 & 11.9 & 49 & 34 & 1.02 & 0.8 \\
\hline & 13.800 & 11.100 & 14 & 12.7 & 39.2 & 37.4 & 1.45 & 0.89 \\
\hline & 21.300 & 15.600 & 15.9 & 14.3 & 45.9 & 41.6 & 0.74 & 0.83 \\
\hline & 18.100 & 13.400 & 14.7 & 12.3 & 41.4 & 34.7 & 0.76 & 0.65 \\
\hline \multirow[t]{2}{*}{ Case report } & 16.900 & 8.330 & 17.4 & 11.4 & 47.6 & 35.2 & 0.91 & 0.78 \\
\hline & 17.300 & 6.230 & 15.9 & 9.86 & 47 & 29 & 1.13 & 0.63 \\
\hline OHSS I (range) & $8.800-29.800$ & $6.300-17.200$ & $12.7-18.1$ & $9.86-14.3$ & $36-51.2$ & $28.9-37.4$ & $0.62-1.45$ & $0.54-0.89$ \\
\hline OHSS II (range) & $13.100-23.400$ & $6.300-17.200$ & $14.5-17.4$ & $11.4-13.2$ & $42-51.2$ & $33.7-37$ & $0.62-1$ & $0.54-0.78$ \\
\hline OHSS III (range) & $9.600-29.800$ & $6.230-15.600$ & $13.3-18.1$ & $10.3-14.3$ & $37.7-49.8$ & 28.9-41.6 & $0.66-1.45$ & $0.54-0.89$ \\
\hline Mean & 16.584 & 10.518 & 15 & 12 & 44 & 35 & 1 & 1 \\
\hline$S D$ & 4940.115064 & 2887.335525 & 1.519233 & 1.039580 & 4.257851 & 2.959354 & 0.222769 & 0.120888 \\
\hline$p$ & \multicolumn{2}{|c|}{$>.0001$} & \multicolumn{2}{|c|}{$>.0001$} & \multicolumn{2}{|c|}{$>.0001$} & \multicolumn{2}{|c|}{$>.0001$} \\
\hline
\end{tabular}

Note: $\mathrm{OHSS}=$ ovarian hyperstimulation syndrome; $\mathrm{x}=$ missing values. $\mathrm{I}=$ mild $\mathrm{OHSS} ; \mathrm{II}=$ moderate $\mathrm{OHSS}$; III = severe OHSS.

history of thromboembolism (the patient's father had a venous thrombosis under the age of 40 and another venous thrombosis, a heart attack and a pulmonary thromboembolic event later in life), although she herself had no evidence of thrombophilic markers. Both pleural and pelvic ascites were evident. The ovaries were enlarged. Hematocrit, CRP, and leukocyte levels were all elevated. Platelets, sodium, potassium, aspartate aminotransferase (AST), alanine aminotransferase (ALT), gamma-glutamyl transferase $(\gamma \mathrm{GT})$ and creatinine were in the normal ranges, whereas albumin and protein were low.

She received the standard treatment, consisting of intravenous hydration with crystalloids and hydroxyethyl starch, anticoagulation, and monitoring of urine output. Both increasing hCG values and transvaginal ultrasonography were indicative of an intrauterine twin pregnancy. Further ultrasound examination showed declining ascites and pleural effusions. The patient felt well and was discharged 10 days after admission. She received anticoagulation treatment with LMWH for another week.
One day after the patient stopped taking her anticoagulation medication, she presented again with a thrombosis of the internal jugular vein and was treated with $\mathrm{LMWH}$ (dalteparin) for anticoagulation, at $2 \times 7500 \mathrm{IU} / \mathrm{d}$. The diagnostic work-up for thrombophilia and antiphospholipid antibodies showed a heterozygotic mutation in the MTHFR gene (C677T) and a lowered protein $S$ value, but an additional test 1 week later showed that protein $S$ was in the normal range. Diamniotic-dichorionic twins with a regular heartbeat were seen on ultrasound. The patient was transferred to the intensive care unit for further treatment and monitoring.

Despite 11 days of therapeutic anticoagulation with unfractionated heparin (UFH) and LMWH, the thrombosis extended to the right subclavian vein, and a new thrombosis occurred in the left jugular vein. Unexpectedly high doses of UFH were needed to achieve therapeutic ranges in the activated partial thromboplastin time. A weight-adjusted dose regimen of nadroparin resulted in subtherapeutic anti-Xa levels 4 hours after subcutaneous injection. Intravenous injection of nadroparin resulted in similar anti-Xa 
Table 4

Outcome of Twin Pregnancies Complicated by Ovarian Hyperstimulation Syndrome

\begin{tabular}{llll} 
& I & II & III \\
\hline Preterm delivery & 1 & 2 & 4 \\
Cesarean section & 1 & 3 & 2 \\
Preterm delivery by cesarean section & 1 & 1 & 2 \\
\hline
\end{tabular}

Note: I = mild ovarian hyperstimulation syndrome (OHSS); II = moderate OHSS; III = severe OHSS.

levels. Therapeutic anticoagulation treatment with UFH was therefore reinstituted on day 12. In addition to the worsening thrombosis, the patient developed a Clostridium difficile infection with diarrhoea.

Eighteen days after the first thrombosis, further treatment options were discussed with the patient. Continuation of the pregnancy with therapeutic anticoagulation rather than pregnancy interruption was recommended, but it was not possible to rule out further risks, including additional thromboembolic incidents. The patient decided in favor of abortion. In the 9th week of gestation (gestational week $8+2$ ), abortion was induced with misoprostol (Cytotec 200) vaginally, followed by curettage. A new thrombolic event was ruled out with Doppler ultrasonography when the patient complained of pain in the left leg. The patient received oral anticoagulation (coumarin) for a further 6 months.

Six months later, she was still suffering pain in the right arm. A Doppler ultrasound examination showed that the right jugular and subclavian vein were still completely obliterated. Six months later, oral anticoagulation treatment was stopped, and the patient's heparin turnover was checked by measuring serum anti-Xa. After administration of a weight-adapted dose of LMWH (0.3), the anti-Xa level 4 hours later was $0.35 \mathrm{U} / \mathrm{ml}$ (within the normal range), in contrast to the values during OHSS.

She was one of two patients with a positive family history of deep vein thrombosis and was the only patient in whom deep vein thrombosis occurred as a complication of OHSS. No further differences from the other patients were observed (Tables 5 and 6).

\section{$\overline{\text { Discussion }}$}

In the patients with OHSS in twin pregnancies reported here, there was a tendency toward more severe forms of OHSS rather than mild or moderate forms. Moreover, the frequency of late OHSS was more than twice that of early OHSS.

Mathur et al. (2000) investigated the association between pregnancy after IVF/ICSI and OHSS. The authors reported that IVF/ICSI pregnancies were more often associated with late OHSS (with an onset more than 10 days after oocyte retrieval) and with severe OHSS. Multiple pregnancies were significantly more frequent in late OHSS. The authors concluded that as
hCG levels tend to be higher in multiple pregnancies from a very early gestational stage, as a result of implantation, the severity of OHSS may reflect a greater magnitude and longer duration of granulosacell stimulation in late OHSS as a result of its association with pregnancy. Polycystic ovaries, estradiol values, and oocyte numbers indicated a risk of early OHSS, whereas these parameters were not predictive of late OHSS. Ascites was observed in almost all patients, dyspnoea in $52.6 \%$, nausea in $36.8 \%$, abdominal pain in $73.6 \%$, pleural effusions in $57.9 \%$, and oedema in $15.8 \%$. Abramov et al. (1998) reported ascites in $98.8 \%$, dyspnoea in $95.7 \%$, gastrointestinal disturbances in $57.7 \%$, pleural effusion in $19 \%$, and oedema in $13.5 \%$ of patients with severe OHSS.

Twin pregnancies are associated with a risk of thromboembolism; one of the 19 patients in the present study developed a thrombosis $(5.2 \%)$. Abramov et al. (1998) reported thromboembolism in $2.5 \%$ of their study population, with one case of induced abortion $(1.5 \%)$ due to deterioration of respiratory status and oliguria. In their series, antenatal complications were more common in patients with severe OHSS in comparison with IVF/ICSI pregnancies without OHSS and included pregnancy-induced hypertension, gestational

\section{Table 5}

Risk Factors for Ovarian Hyperstimulation Syndrome and

Thromboembolism in the Patient Described in the Case Report

Risk factors

Father with a history of thromboembolism

Heterozygotic MTHFR mutation (C677T)

Young age

Twin pregnancy

Ovulation induction with hCG

Luteal support with hCG

$>15$ oocytes retrieved

Note: $\mathrm{hCG}=$ human chorionic gonadotrophin.

\section{Table 6}

Course of Parameters in the Patient Described in the Case Report

\begin{tabular}{lccccc}
\hline Course of parameters & & & & & \\
\hline $\begin{array}{l}\text { Day after ovulation } \\
\text { induction }\end{array}$ & 14 & 16 & 19 & 21 & 23 \\
Leukocytes & 16.9 & 12.0 & 9.4 & 9.9 & 8.3 \\
Platelets & 311 & 222 & 299 & 263 & 292 \\
Hematocrit & 47.6 & 40.5 & 35.3 & 34.4 & 35.2 \\
Estradiol & 4967 & 3815 & 5101 & $\mathrm{x}$ & 4917 \\
Progesterone & $>400$ & 229 & 244 & $\mathrm{x}$ & 226 \\
hCG & 76 & 159 & 510 & $\mathrm{x}$ & 2513 \\
Albumin & 33.5 & 27.4 & $\mathrm{x}$ & 32.1 & $\mathrm{x}$ \\
Creatinine & $\mathrm{x}$ & $\mathrm{x}$ & $\mathrm{x}$ & 0.76 & $\mathrm{x}$ \\
\hline
\end{tabular}

Note: $h C G=$ human chorionic gonadotrophin; $x=$ missing values. 
diabetes, late intrauterine fetal demise, preterm rupture of membranes, placental abruption, and low birthweight. The authors concluded that antenatal complications in IVF/ICSI pregnancies with OHSS occur significantly more frequently than in IVF/ICSI pregnancies without OHSS. Patients with severe OHSS are exposed to high endogenous oestrogen levels, in addition to cytokines, renin, angiotensin, and possibly prostaglandins, with an unknown effect on early pregnancies. However, these mechanisms do not increase the prevalence of fetal malformations after severe OHSS.

Wiser et al. (2005) compared twin pregnancies after IVF/ICSI complicated by OHSS with twin pregnancies after IVF/ICSI without OHSS. In 64 twin pregnancies with OHSS, the patients were significantly younger and had polycystic ovaries significantly more often than in the control group without OHSS. There were no differences in the incidence of gestational diabetes mellitus, hypertensive disorders, gestational week at delivery, birthweight, or rate of cesarean section. In comparison with singleton pregnancies with OHSS, there was a significantly longer hospitalization period and a higher risk of ovarian torsion in twin pregnancies $(10.9 \%$ vs. $3 \%)$.

In the patients included in the present study, two women with OHSS and twin pregnancies reported bleeding in early pregnancy $(10.5 \%)$. The values and ranges for leukocytes, hemoglobin, hematocrit, platelets, estradiol, progesterone, and even hCG in patients with twin pregnancies in the present study were comparable to those in patients with singletons.

Pinborg (2005) reported similar risks in IVF/ICSI and control twins with regard to birthweight, gestational age, and the incidence of small-for-gestational-age children. Cesarean section rates, including the frequency of emergency cesarean operations and the rate of vacuum extractions, were higher in IVF/ICSI twin pregnancies than in twin pregnancies without assisted reproduction techniques. It is likely that the slightly higher risk of neonatal intensive care unit admittance in IVF/ICSI twins is due to greater precautions being taken in the highly valued IVF/ICSI twin pregnancies.

The risk of developing a thrombosis during pregnancy is 20 to 40 times lower than the risk of OHSS combined with a pregnancy (Ray \& Chan, 1999). Venous thromboembolisms in pregnancy are usually located in the lower extremities, with $70 \%$ of cases occurring in the ileofemoral region (Kyrle \& Eichinger, 2005). In OHSS patients, however, $60 \%$ of thromboembolic events in the 97 cases reported in the literature to date were located in the upper part of the body, with $67 \%$ to $75 \%$ being venous and $25 \%$ to $33 \%$ arterial. Thromboembolism in the jugular vein occurs on average $43 \pm 30$ days after ovulation induction (Delvigne \& Rozenberg, 2003). In the patient described above, the first thrombosis occurred on day 31 after the induction of ovulation with hCG. It is not known why OHSS appears to lead to a predilection for thromboembolism developing in the upper part of the body. In a total of 21 cases reported up to 2003, only two patients developed a bilateral jugular vein thrombosis (Delvigne \& Rozenberg, 2003). In cases of thrombosis, $85 \%$ of the affected patients were pregnant and $75 \%$ had OHSS. Hypercoagulopathy correlates positively with high estrogen levels, immobilization, high hematocrit levels, increased intra-abdominal pressure (ascites, enlarged ovaries) and lowered tone in the venous system due to high levels of progesterone in pregnant OHSS patients (Pinborg, 2005; Pinborg et al., 2004). The incidence is therefore likely to be even higher in twin pregnancies with OHSS.

Eighty-five per cent of patients with OHSS have one or more elevated markers of thrombophilia (Binder et al., 2004). Rao et al. (2005) reported that a patient with a jugular vein thrombosis had a possible protein $\mathrm{S}$ deficiency, but no family history of thromboembolism, whereas in the patient described here, there was a family history of thromboembolism and a temporary decrease in protein $\mathrm{S}$, combined with a heterozygous C677T mutation.

Unfortunately, thrombosis may occur despite appropriate management and even when prophylactic anticoagulation is administered (Hignett et al., 1995). This may result from heparin-induced antibodies leading to a highly specific and paradoxical form of thrombocytopenia that promotes thrombosis rather than bleeding. Data from pregnant women without OHSS (Barbour et al., 2004; Ensom \& Stephenson, 2004; Sephton et al., 2003) show that the pharmacokinetics of LMWH are markedly altered, resulting in lower peak and trough levels. This may lead to treatment failures in pregnancy, with or without OHSS. Close monitoring of anti-Xa levels and thrombus progression can therefore be recommended. In patients who experience thrombotic events despite adequate anticoagulation therapy, heparin-induced thrombocytopenia should be ruled out.

\section{$\overline{\text { References }}$}

Abramov, Y., Elchalal, U., \& Schenker, J. G. (1998). Obstetric outcome of in vitro fertilized pregnancies complicated by severe ovarian hyperstimulation syndrome: A multicenter study. Fertility and Sterility, 70, 1070-1076.

Barbour, L. A., Oja, J. L., \& Schultz, L. K. (2004). A prospective trial that demonstrates that dalteparin requirements increase in pregnancy to maintain therapeutic levels of anticoagulation. American Journal of Obstetrics and Gynecology, 191, 1024-1029.

Binder, H., Cupisti, S., Dittrich, R., Einhaus, F., Krieg, J., Müller, A., Strauss, R., \& Beckmann, M. W. (2004). GebFra-Refresher: Ovarielles Überstimulationssyndrome [Ovarian hyperstimulation syndrome Update Part 1 and 2]. Geburtshilfe und Frauenheilkunde, 64, R53-100.

Delvigne, A., \& Rozenberg, S. (2003). Review of clinical course and treatment of ovarian hyperstimulation 
syndrome (OHSS). Human Reproduction Update, 9, 77-96.

Ensom, M. H., \& Stephenson, M. D. (2004). Pharmacokinetics of low molecular weight heparin and unfractionated heparin in pregnancy. Journal of the Society for Gynecologic Investigation, 11, 377-383.

Hignett, M., Spence, J. E., \& Claman, P. (1995). Internal jugular vein thrombosis: A late complication of ovarian hyperstimulation syndrome despite mini-dose heparin prophylaxis. Human Reproduction, 10, 3121-3123.

Kyrle, P. A., \& Eichinger, S. (2005). Deep vein thrombosis. Lancet, 365, 1163-1174.

Mathur, R. S., Akande, A. V., Keay, S. D., Hunt, L. P., \& Jenkins, J. M. (2000). Distinction between early and late ovarian hyperstimulation syndrome. Fertility and Sterility, 73, 901-907.

Pinborg, A. (2005). IVF/ICSI twin pregnancies: Risks and prevention. Human Reproduction Update, 11, 575-593.

Pinborg, A., Loft, A., \& Nyboe Andersen, A. (2004). Neonatal outcome in a Danish national cohort of
8602 children born after in vitro fertilization or intracytoplasmic sperm injection: The role of twin pregnancy. Acta Obstetricia et Gynecologica Scandinavica, 83, 1071-1078.

Rao, A. K., Chitkara, U., \& Milki, A. A. (2005). Subclavian vein thrombosis following IVF and ovarian hyperstimulation: A case report. Human Reproduction, 20, 3307-3312.

Ray, J. G, \& Chan, W. S. (1999). Deep vein thrombosis during pregnancy and the puerperium: A meta-analysis of the period of risk and the leg of presentation. Obstetrical and Gynecological Survey, 54, 265-271.

Sephton, V., Farquharson, R. G., Topping, J., Quenby, S. M., Cowan, C., Back, D. J., \& Toh, C. H. (2003). A longitudinal study of maternal dose response to low molecular weight heparin in pregnancy. Obstetrics and Gynecology, 101, 1307-1311.

Wiser, A., Levron, J., Kreizer, D., Achiron, R., Shrim, A., Schiff, E., Dor, J., \& Shulman, A. (2005). Outcome of pregnancies complicated by severe ovarian hyperstimulation syndrome (OHSS): A follow-up beyond the second trimester. Human Reproduction, 20, 910-914. 